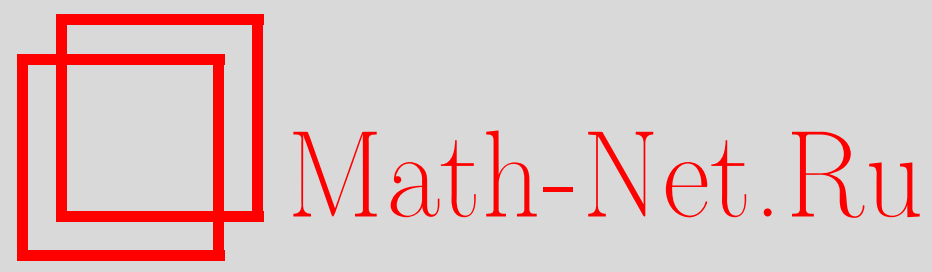

А. А. Азамов, Х. К. Яхшимов, Задача качества для одной дифференциальной игры с критическими свойствами, $M a-$ тем. заметки, 2000, том 67, выпуск 4, 484-488

DOI: https://doi.org/10.4213/mzm862

Использование Общероссийского математического портала Math-Net.Ru подразумевает, что вы прочитали и согласны с пользовательским соглашением http://www.mathnet.ru/rus/agreement

Параметры загрузки:

IP : 54.209 .52 .79

26 апреля 2023 г., 05:31:29

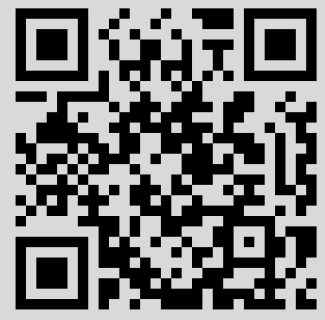




\title{
ЗАДАЧА КАЧЕСТВА ДЛЯ ОДНОЙ ЛИНЕЙНОЙ ДИФФЕРЕНЦИАЛЬНОЙ ИГРЫ С КРИТИЧЕСКИМИ СВОЙСТВАМИ
}

\author{
А. А. Азамов, Х.К. Яхшимов
}

В работе приводится решение задачи преследования для одной линейной дифференциальной игры, являющейся критической - лежащей на границе разрешимости задач сближения и уклонения. На основе полученного результата дается ответ на два вопроса, связанных с методами Понтрягина.

Библиография: 11 названий.

1. Один из простых, но нетривиальных примеров теории дифференциальных игр задается системой

$$
\dot{x}=-y-u_{1}+v_{1}, \quad \dot{y}=x-u_{2},
$$

терминальным множеством $M=\{0\}$ и областями управлений преследователя $P=H$ и убегающего $Q=[-1,1]$ соответственно. (Всюду $r H$ обозначает круг $|z| \leqslant r$, где $z=(x, y)$.) Содержание игры состоит в том, что преследователь, управляющий вектором $u, u \in P$, стремится привести фазовую точку из начального состояния $\xi=\left(x_{0}, y_{0}\right)$ в точку 0 , второй игрок - убегающий, управляющий параметром $v, v \in Q$, имеет противоположную цель. (В дальнейшем $\xi \neq 0$ и $\bar{Q}$ обозначает $Q \times\{0\}$.)

Отметим примечательную особенность примера (1). С одной стороны, $\bar{Q} \subset P$, причем $\bar{Q} \neq P$, так что известные теоремы об убегании ([1] и др.) не применимы. С другой стороны, преследователь не имеет явного преимущества, так как $P \stackrel{*}{-} Q=0$. Вследствие этого разрешимость задачи преследования непосредственно не вытекает из первого метода Понтрягина и его модификаций [2]-[4]. Что касается метода характеристик (т. е. эвристического метода Айзекса [5]), методов альтернированного интеграла [6] и стохастического максимина [7], то попытки их применения сопряжены с трудностями аналитического характера.

Критичность игры (1) проявляется и в том, что если круг $P$ заменить квадратом $\{u=$ $\left.\left(u_{1}, u_{2}\right)|| u_{1}|+| u_{2} \mid \leqslant 1\right\}$, то задача преследования становится неразрешимой.

В настоящей работе с помошью альтернированного интеграла доказьвается, что если существует хотя бы одно начальное положение, из которого возможно завершение преследования, то это имеет место для любого начального положения. Затем для точки $\xi=(\sqrt{2} / 8,1 / 4)$ строится специальная стратегия преследования, позволяющая привести фазовую точку в 0 вдоль “поводыря" - кривой $2 y^{3}=x^{2}$. 
2. ОПРЕДЕЛЕНИЯ. Позиционной контрстратегией преследователя (короче cтратегией) $U$ назьвается функция вида $u(t, z, v)$, отображающая $[0, \theta] \times \mathbb{R}^{2} \times Q$ в множество $P$ и являющаяся измеримой функцией $t$ при любых фиксированных $z, v$ и измеримой по Борелю по $v$ при любых фиксированных $t$ и $z$. Управлением убегающего называется произвольная измеримая функция $v(\cdot):[0, \theta] \rightarrow Q$. Каждая тройка, состоящая из начальной точки $\xi$, стратегии $U$ и управления $v(\cdot)$, порождает пучок $m p a-$ екторий $Z(\xi, U, v(\cdot))$, которые определяются как равномерныепределы ломаных Эйлеpa [7], [8]. Далее, по определению для начальной точки $\xi$ задача сближения на отрезке $[0, \theta]$ разрешима, если существует стратегия преследователя $U^{*}$ такая, что при любом управлении убегающего $v(\cdot)$ для каждой траектории $z(\cdot) \in Z\left(\xi, U^{*}, v(\cdot)\right)$ имеет место равенство $z(t)=0$ при некотором $t \in[0, \theta]$.

Игра (1) имеет ряд полезных специфических качеств.

ПРЕДЛОЖЕНИЕ 1. Если для некоторой начальной точки $\xi$ разрешима задача сближения на отрезке $[0, T]$, то она разрешима и для всех точек $\eta \in|\xi| H$ на отрезке $[0, T+\pi]$.

ДокАЗАТЕЛЬСТво. Пусть $U_{\xi}=u_{\xi}(\cdot, \cdot, \cdot)$ - стратегия, обеспечивающая решение задачи сближения для начальной точки $\xi$ на отрезке $[0, T]$. Тогда ввиду симметричности игры (1) относительно точки 0 стратегия $-u_{\xi}(t,-z, v)$ гарантирует разрешимость задачи сближения для точки $-\xi$.

Пусть $\eta$ - произвольная точка окружности радиуса $|\xi|$ с центром 0 и для определенности $\eta$ получается поворотом $\xi$ вокруг точки 0 на угол $-\varphi, 0<\varphi<\pi$. Определим стратегию $U_{\eta}=u_{\eta}(\cdot, \cdot, \cdot)$ следуюшим образом:

$$
\begin{array}{ll}
u_{\eta}(t, z, v)=(v, 0) & \text { при } 0 \leqslant t<\varphi, \\
u_{\eta}(t, z, v)=u_{\xi}(t-\varphi, z, v) & \text { при } \varphi \leqslant t .
\end{array}
$$

Тогда при любом управлении $v(\cdot)$ сужение пучка $Z(\eta, U \eta, v(\cdot))$ на отрезок $[0, \varphi]$ состоит из одной функции $z_{0}(t)$ такой, что $z_{0}(\varphi)=\xi$. Поэтому для любой траектории $z(\cdot) \in Z\left(\eta, U_{\eta}, v(\cdot)\right)$ имеет место $z(t)=0$ при некотором $t, t \leqslant T+\varphi<T+\pi$.

Пусть теперь $|\eta|<|\xi|$. Чтобы убедиться в том, что для точки $\eta$ разрешима задача сближения на отрезке $[0, T+\pi]$, достаточно заметить следующее свойство. Пусть $v(\cdot)$ - фиксированное управление убегающего, $z(\cdot)$ - траектория из пучка $Z\left(\xi, U_{\xi}, v(\cdot)\right)$, так что $z\left(t^{*}\right)=0$, где $t^{*} \in[0, T]$. Тогда для каждой точки $\eta=z(t), 0<t \leqslant t^{*}$ (как начальной), задача сближения разрешима на отрезке $\left[0, T-t^{*}\right]$. Если точка $\eta$ не лежит на дуге $z(t), 0<t \leqslant \pi$, то учитьвая, что при $u=(v, 0)$ фазовая точка движется по окружности, легко построить стратегию, обеспечивающую разрешимость задачи сближения из начального состояния $\eta$ на отрезке $[0, T+\pi]$.

ПРЕДЛОЖЕНИЕ 2. Если для Каждой начальной точки из круга $l_{0} H$ заданного радиуса $l_{0}, l_{0}>0$, разрешима задача сближения на фиксированном отрезке $[0, T]$, то она разрешима и для любой точки $\xi \in \mathbb{R}^{2}$ на некотором отрезке.

ДоКАЗАТЕЛЬСТво можно проводить непосредственно - построением в явном виде стратегии преследователя, приводящей пучок траекторий на круг $l_{0} H$. Однако это сопряжено с громоздкими вычислениями. Один элемент такой стратегии использован ниже - при доказательстве предложения 3. Здесь предложение 2 доказьвается применением альтернативного интеграла [6]. При этом без потери общности считается $l_{0} \leqslant 2$. 
Рассмотрим дифференциальную игру (1) с новым терминальным множеством $M=l H$. Альтернативный интеграл $W^{\tau}(l H)$ также не удается вычислить в явном виде. Выведем оценку снизу. Известно [6], что $W^{\tau}(M) \supset\left(M^{*}-B\right)+A$, где

$$
A=\int_{0}^{\tau} e^{r C} P d r, \quad B=\int_{0}^{\tau} e^{r C} Q d r
$$

$C-(2 \times 2)$-матрица коэффищиентов системы (1). Поскольку $e^{r C}$-матрища поворота, то $A=\tau H$. При $\tau \leqslant \pi$ имеет место соотношение $B \subset 2 \sin (\tau / 2) H$ [9]. Поэтому

$$
W^{\tau}(l H) \supset\left(l+\tau-2 \sin \left(\frac{\tau}{2}\right)\right) H \quad \text { для } \tau \in[0, l] .
$$

В частности,

$$
W^{l}(l H) \supset\left(l+\frac{l^{3}}{32}\right) H \quad(l \leqslant \pi) .
$$

Пусть последовательность $l_{n}$ задана формулой $l_{n+1}=l_{n}+l_{n}^{3} / 32$. Положим $\sigma_{n}=$ $l_{0}+l_{1}+\cdots+l_{n}$. Тогда в силу полугрупшового свойства альтернативного интеграла [6] и соотношения (3):

$$
W^{\sigma_{1}}\left(l_{0} H\right)=W^{l_{1}}\left(W^{l_{0}}\left(l_{0} H\right)\right) \supset W^{l_{1}}\left(l_{1} H\right) \supset l_{2} H .
$$

Аналогично,

$$
W^{\sigma_{n}}\left(l_{0} H\right) \supset l_{n+1} H .
$$

Поскольку последовательность $l_{n}$ возрастает, то $l_{n} \geqslant l_{0}+l_{0}^{3} / 32$ пока $l_{n} \leqslant \pi$. Очевидно, сушествует $k$ такое, что $2 \leqslant l_{k+1}<\pi$. Если $k$ наименьшее среди них, то положим $m=\sigma_{k}$. Тогда

$$
W^{m}\left(l_{0} H\right) \supset 2 H .
$$

Далее, пользуясь оценкой (4) и формулой

$$
\int_{0}^{n \pi} e^{r C} \bar{Q} d r=2 n H
$$

(см. [9]), легко вывести соотношение

$$
W^{n \pi+m}\left(l_{0} H\right) \supset W^{n \pi}(2 H) \supset 2(n+1) H .
$$

Из него вытекает, что при $\tau \rightarrow \infty$ альтернированньй интеграл $W^{\tau}\left(l_{0} H\right)$ покрьвает всю плоскость $\mathbb{R}^{2}$.

Для завершения доказательства остается заметить, что точки $(\tau, z)$, для которых $e^{\tau C} z \in W^{\tau}\left(l_{0} H\right)$, образуют $u$-стабильньй мост [8]. Поэтому для любой начальной точки $\xi$ задача сближения (с терминальным множеством $l_{0} H$ ) разрешима на отрезке $[0, T]$, где $T \leqslant \pi[|\xi| / 2]+m$.

3. Из предложений 1 и 2 вытекает, что для игры (1) альтернатива имеет место в усиленной форме: если сушествует хотя бы одна начальная точка, для которой разрешима задача сближения на некотором отрезке, то это имеет место для любой начальной точки. 
ПРЕДЛОЖЕНИЕ 3. Для начальной точки $x_{0}=\sqrt{2} / 8, y_{0}=1 / 4$ задача сближения разрешима на отрезке $[0,10]$.

ДокАЗАтЕльство. Рассмотрим стратегию преследователя $u^{*}(z, v)=\left(u_{1}^{*}, u_{2}^{*}\right)$, заданную в достаточно малой окрестности дуги $\Delta=\left\{(x, y) \mid 0 \leqslant x \leqslant x_{0}, 2 y^{3}=x^{2}\right\}$ формулами:

$$
u_{1}^{*}=\frac{A x+3 B y^{2}}{R}, \quad u_{2}^{*}=\frac{B x-3 A y^{2}}{R},
$$

где $A=v x-3 x y^{2}-x y, B=\left(9 y^{4}+x^{2}-A^{2}\right)^{1 / 2}, R=A^{2}+B^{2}$. (Значения функции $u^{*}$ вне малой окрестности $\Delta$ здесь не играет роли.)

Задача Коши $x(0)=x_{0}, y(0)=y_{0}$ для системы $(1)$, в которой положено $u=u^{*}(z, y(t))$, $v=v(t)$ - произвольное управление убегающего, удовлетворяет условиям теоремы существования и единственности и имеет первьй интеграл $2 y^{3}=x^{2}$. Таким образом, точка $z(t)$ скользит по дуге $\Delta$. Остается показать существование момента времени $t^{*} \in[0,10]$ такого, что $y(t) \rightarrow 0$ при $t \rightarrow t^{*}$.

Выражение $x-u_{2}^{*}(z, v)$ для производной $\dot{y}$ (см. (1)) достигает максимум по $v \in Q$ при $v=1$. Учитьвая это обстоятельство и наличие первого интеграла, пользуясь неравенством $1-3 y^{2}-y \geqslant\left(1-3 y^{2}-y\right)^{2}$, имеющим место при $0 \leqslant y \leqslant y_{0}$, можно вывести оценку

$$
\dot{y} \leqslant \frac{\sqrt{2 y}}{9 y+2}(3-y-\sqrt{6 y+11}) \leqslant \frac{\sqrt{2 y}(3-\sqrt{11})}{9 y+2} .
$$

Отсюда следует, что $\dot{y} \leqslant 0$ пока $y \geqslant 0$, и следовательно, $y \leqslant y_{0}=1 / 4$. Далее, неравенство (5) влечет соотношение

$$
\dot{y} \leqslant-4(\sqrt{11}-3) \frac{\sqrt{2 y}}{17} \leqslant-0.1 \sqrt{y}
$$

откуда вытекает требуемое.

СлЕДСТВИЕ 1. В игре (1) для каждой начальной точки $\xi \in \mathbb{R}^{2}$ разрешима задача сближения на конечном отрезке времени.

СлЕДСТВИЕ 2. Для дифференииальной игры (1) при $\tau>0$ альтернированный интеграл $W^{\tau}(\{0\})$ содержит круг положительного радиуса $r$, причем $r \rightarrow \infty$ при $\tau \rightarrow \infty$.

4. Следствие 2 дает ответ на два вопроса об альтернированном интеграле Понтрягина. Один из них относится к сравнению двух методов преследования [6]. Было предложено несколько усилений первого метода. Последнее из них [4] во всех известных примерах давало тот же результат, что и второй метод. Если интеграл, введенньй в работе [4], обозначить через $W_{4}^{\tau}(M)$, то из общей теории вытекает, что $W_{4}^{\tau}(M) \subset W^{\tau}(M)$. В связи с этим возник вопрос о том, имеет ли место равенство $W_{4}^{\tau}(M)=W^{\tau}(M)$. Для дифференциальной игры (1) $W_{4}^{\tau}(M)=0$. Следовательно, метод альтернированного интеграла сильнее всех известных вариантов первого (прямого) метода.

Второй вопрос связан с работой [10]. В ней построен интеграл $\widetilde{W}^{\tau}(M)$, отличающийся от интеграла $W^{\tau}(M)$ тем, что при образовании альтернированных сумм $\widetilde{S}^{\omega}(M)$ интегралы (2) заменяются их средними значениями. В работе [10] доказано, что

$$
\widetilde{W}^{\tau}(M) \subset W^{\tau}(M) \bigcap_{\omega} \widetilde{S}^{\omega}(M+\gamma(|\omega|) H),
$$


где $\gamma(t) \rightarrow 0$ при $t \rightarrow 0$.

Следствие 2 показывает, что надевание “шапок Никольского" $\gamma(|\omega|) H$ на терминальное множество $M$ является существенным для того, чтобы имело место правое равенство (6), потому что для игры (1) выполняется условие $\widetilde{W}^{\tau}(M)=0$. Это заключение сохраняет силу и для других аналогичных упрощенных интегралов, введенных в работе [11].

\section{СПИСОК ЦИТИРОВАННОЙ ЛИТЕРАТУРЫ}

[1] Понтрягин Л. С. Линейная дифференциальная игра убегания // Тр. МИАН. 1971. Т. 112. C. $30-63$.

[2] Никольский М.С. Об одном прямом методе решения линейных дифференциальных игр преследования-убегания // Матем. заметки. 1983. Т. 33. №6. С. 885-891.

[3] Азамов А. А., Саматов Б. Т. О модифицированном третьем методе в задачах преследования // Неклассические задачи математической физики. Ташкент: Фан, 1985. С. 174-184.

[4] Сатимов Н., Карабаев Э. О. Об одном методе решения задачи преследования // Докл. АН У ${ }_{3}$ ССР. 1986. № 3. С. 7-9.

[5] Айзекс Р. Дифференциальные игры. М.: Наука, 1967.

[6] Понтрягин Л.С. Линейные дифференциальные игры преследования // Матем. сб. 1980. T. 112. №3. C. 307-330.

[7] Красовский Н. Н. Управление динамической системой. М.: Наука, 1985.

[8] Субботин А. И., Ченцов А. Г. Оптимизация гарантии в задачах управления. М.: Наука, 1981.

[9] Абдуганиев А. А., Исканаджиев И. М. О задаче вычисления альтернированного интеграла Понтрягина // Докл. АН УзССР. 1985. № 7. С. 5-6.

[10] Никольский М. С. Об альтернированном интеграле Л. С. Понтрягина // Матем. сб. 1981. T. 116. № 4. C. 136-144.

[11] Азамов А. А. Двойственность и полуустойчивость альтернированного интеграла // Докл. AH CCCP. 1988. T. 299. № 2. C. 265-268.

Наманганский государственный университет 